\title{
472 - Santa Isabel Program for psycho-motor function in normal and pathological aging during current times of COVID-19 confinement in a long-term care
}

Lidia Castillo-Mariqueo $^{1,2,3}$ Patricia Valenzuela-Garrido ${ }^{4}$ and Lydia Giménez-Llort ${ }^{1,2,3}$

${ }^{1}$ Department of Psychiatry and Forensic Medicine, School of Medicine, Universitat Autònoma de Barcelona, Barcelona, Spain

${ }^{2}$ Institut de Neurociències, Universitat Autònoma de Barcelona, Barcelona, Spain

${ }^{3}$ Envellir bé - Saber Envejecer - Healthy Aging Charity Organization, Sant Quirze del Vallès, Barcelona, Spain

${ }^{4}$ Establecimiento de Larga Estadía para el Adulto Mayor de Santa Isabel, Traiguén - Club Leones, Chile

Since its appearance, the COVID-19 pandemic has generated a state of alarm worldwide. Extraordinary confinement measures, only seen in times of war, have been implemented to halt the fast viral transmission, the consequent stress and overload of health systems and, mostly, the dead of the most vulnerable people. Physical and social isolation has changed the lifestyle we are used to, affecting our mood and mental health at a general level. However, the group of greatest risk and vulnerability are the elder age group, due to their higher incidence of frailty, their immunosenescence and comorbidities. Also, in this elder group of the population, the consequences of social isolation may be worse to handle, especially in those who live alone and do not have a family or a strong community bond. In the case of long-term institutionalized patients, these clinical scenarios are struggling to maintain their rehabilitation and care programs while desperately adapting to the dramatic situations due to the severe impact of COVID-19 in these care settings. To address this complex scenario, we developed and implemented the Santa Isabel Program, a series of recommendations for long-term care with the aim of guiding and promoting routines, activities and habits to help maintenance of psycho-motor function of older people during the state of alarm and thereafter. The Santa Isabel Program was defined as a general therapeutic resource with the selection of different modalities of physical activity (daily walk, adapted gymnastics and dance) and functional cognitive activity (meditation, relaxation, memory and attention). Chronogram, time and intensity levels were of adjustable dosage to respond to the needs and requirements of the elder people according to the severity and type of physical or cognitive impairment. In addition, specific indications were developed for cases of people with cognitive pathology (dementia). These guidelines were prepared for the professional team and support staff, in order to provide tools that allow them to resolve complex situations such as BPSD, the behavioral and psychological symptoms of dementia. Finally, audiovisual support sessions and video calls were incorporated to allow interaction with residents and the health team, safeguarding the COVID-19 infection prevention measures. 